\title{
Preface
}

\section{MaCWAVE: a rocket-lidar-radar program to study the polar mesosphere during summer and winter}

\author{
D. C. Fritts ${ }^{1}$ and R. A. Goldberg ${ }^{2}$ \\ ${ }^{1}$ Northwest Research Associates, Colorado Research Associates Division, Boulder, Colorado, CO, USA \\ ${ }^{2}$ NASA/Goddard Space Flight Center, Greenbelt, Maryland, USA
}

The MaCWAVE (Mountain and Convective Waves Ascending VErtically) coordinated rocket, ground-based, and satellite measurement program was designed to study gravity wave dynamics and forcing of the mesosphere and lower thermosphere (MLT) during typical summer and winter conditions. The summer MaCWAVE program was performed at the Andøya Rocket Range (ARR, 69.3 $\mathrm{N}$ ) in northern Norway during July 2002. The winter program was performed at Esrange $\left(67.9^{\circ} \mathrm{N}\right)$ in northern Sweden during January 2003. Neither program, however, occurred under typical solstice conditions. Instead, each program revealed considerably greater variability in gravity wave, and largerscale, dynamics and their influences than had been seen previously at these latitudes. Indeed, the MaCWAVE program appears to comprise the only suite of comprehensive measurements throughout the lower and middle atmosphere at polar latitudes during stratospheric warmings, or their largescale precursors, occurring in both hemispheres.

Summer measurements were performed together with the MIDAS (MIddle atmosphere Dynamics And Structure) program designed by German and Norwegian colleagues. Together, the unique MaCWAVE/MIDAS data set includes measurements employing the ALOMAR MF, MST, and meteor radars, ALOMAR RMR and Na lidars, and radiosonde, MET rocket falling spheres, and Terrier-Orion soundings of winds, temperatures, neutral and electron densities, and plasma measurements extending from the surface into the thermosphere. Winter measurements employed the groundbased instrumentation at ALOMAR noted above, as well as a Rayleigh lidar, meteor radar, and all-sky camera at Esrange, and additional radiosondes, falling spheres, and Terrier-Orion payloads launched from Esrange. Several of the fallings spheres during both summer and winter campaigns were launched to coordinate as fully as possible with SABER temperature measurements during TIMED satellite overpasses.
Results of our initial analyses of summer MaCWAVE/MIDAS measurements appeared as an 8paper special section of Geophysical Research Letters in December 2004, and will not be discussed here. The emphasis in this special section of Annales Geophysicae is on subsequent, and more comprehensive, analyses of MaCWAVE data, with a primary focus on our winter measurements from ALOMAR and Esrange. As noted above, winter measurements were intended to address the propagation of gravity waves arising from air flow over the Scandinavian topography into the upper mesosphere and lower thermosphere, where we expected them to achieve large amplitudes and momentum fluxes and to lead to significant instability dynamics and mean flow interactions. However, our measurements coincided with the occurrence of a stratospheric warming that caused significant departures of the large-scale wind and temperature fields from typical winter conditions. Reduced and/or reversed zonal winds in the middle and upper mesosphere resulted in blockage of the penetration of gravity waves arising from the Scandinavian topography to high altitudes. Instead, these waves were found to be trapped and to encounter critical levels at lower altitudes, as expected from linear theory.

There are nine papers, including the initial overview, appearing in this MaCWAVE special issue. These papers span a wide range of topics from the general circulation of the atmosphere and inter-hemispheric coupling during northern hemisphere summer in 2002 to small-scale neutral and plasma dynamics and layering under summer and winter conditions. They provide a general introduction to all of the MaCWAVE results obtained to date, including a summary of results of our previous special issue in Geophysical Research Letters focusing on the summer MaCWAVE/MIDAS measurement program. Successive papers are organized according to the spatial and temporal scales of the processes addressed, from larger to smaller. The papers examine 
the influences of inter-hemispheric coupling arising from anomalously strong planetary wave activity in the southern winter hemisphere on the summer polar MLT circulation and thermal structure, as well as the large-scale environment, including stratospheric warming responses which accompanied the winter MaCWAVE measurement program in January and February 2003. Others examine gravity wave responses and instabilities accompanying large-amplitude tidal motions and propagation into a weak or reversed zonal flow due to the stratospheric warming occurring during our winter measurement program. They also analyze the character of small-scale gravity wave and instability structures near the mesopause in winter. Finally, they examine the plasma domain, to study the microphysics underlying polar mesosphere winter echoes, correlated sporadic sodium and $E$ layers, and plasma structures at small spatial scales.

David C. Fritts and Richard A. Goldberg

Special Issue Editors 